\title{
Activated Hepatic Stellate Cells Induce Infiltration and Formation of CD163+ Macrophages via CCL2/CCR2 Pathway
}

\section{Sujuan Xi}

Department of Infectious Diseases, Third Affiliated Hospital of Sun Yat-sen University, Guangzhou, Guangdong 510630, China. Key Laboratory of Tropical Disease Control (Sun Yat-Sen University), Ministry of Education, Guangzhou, Guangdong 510080, China. The

\section{Xiaoyan Zheng}

Department of Infectious Diseases, Third Affiliated Hospital of Sun Yat-sen University, Guangzhou, Guangdong 510630, China

\section{Yuming Jiang}

Department of General Surgery, Nanfang Hospital, Southern Medical University, 1838 North Guangzhou Avenue, Guangzhou, China

\section{Yuankai Wu}

Department of Infectious Diseases, Third Affiliated Hospital of Sun Yat-sen University, Guangzhou, Guangdong 510630, China

\section{Jiao Gong}

Department of Infectious Diseases, Third Affiliated Hospital of Sun Yat-sen University, Guangzhou, Guangdong 510630, China. Key Laboratory of Tropical Disease Control (Sun Yat-Sen University), Ministry of Education, Guangzhou, Guangdong 510080, China

\section{Yusheng Jie}

Department of Infectious Diseases, Third Affiliated Hospital of Sun Yat-sen University, Guangzhou, Guangdong 510630, China. Key Laboratory of Tropical Disease Control (Sun Yat-Sen University), Ministry of Education, Guangzhou, Guangdong 510080, China

\section{Xiangyong Li}

Department of Infectious Diseases, Third Affiliated Hospital of Sun Yat-sen University, Guangzhou, Guangdong 510630, China

\section{Jing Cao}

Department of Infectious Diseases, Third Affiliated Hospital of Sun Yat-sen University, Guangzhou, Guangdong 510630, China. Key Laboratory of Tropical Disease Control (Sun Yat-Sen University), Ministry of Education, Guangzhou, Guangdong 510080, China

\section{Liuping Sha}

Department of Infectious Diseases, Third Affiliated Hospital of Sun Yat-sen University, Guangzhou, Guangdong 510630, China 


\section{Min Zhang}

Department of Infectious Diseases, Third Affiliated Hospital of Sun Yat-sen University, Guangzhou, Guangdong 510630, China. Key Laboratory of Tropical Disease Control (Sun Yat-Sen University), Ministry of Education, Guangzhou, Guangdong 510080, China

\section{Yutian Chong ( $D$ ytchongkyzy@126.com )}

Third Affiliated Hospital of Sun Yat-Sen University https://orcid.org/0000-0001-8215-4393

\section{Research}

Keywords: Activated hepatic stellate cells, Liver fibrosis, M2 macrophage; CCL2, Hepatitis B.

Posted Date: May 17th, 2020

DOI: https://doi.org/10.21203/rs.3.rs-28626/v1

License: (c) (i) This work is licensed under a Creative Commons Attribution 4.0 International License. Read Full License 


\section{Abstract}

Background: Growing evidence indicates that activated hepatic stellate cells (aHSCs) play unexpected roles in regulating immune cells' function during liver fibrosis. Macrophages feature with inducible plasticity according to the circumstances while patrol for potential pathogens. However, studies seldom investigate whether and how the aHSCs regulate the phenotype and function of macrophages during liver fibrosis.

Methods: 96 patients with different stages of liver fibrosis were involved in our study. Metavir score system was used to evaluate the degree of fibrosis. The expression of hepatic CCL2 and CD163 were detected by immunohistochemistry, and the relationship among CD163, CCL2, and fibrosis scores were explored. We co-cultured the aHSCs and THP-1-derived M0-type macrophages (MOMФ) to observe whether aHSCs modulate the expression of CD163 on macrophages in vitro. Furthermore, we treated the MOMФ with aHSCs' supernatant and investigated the production of CCL2 in aHSCs by ELISA and immunofluorescence assay. To explore whether CCL2/CCR2 axis plays a crucial role in macrophage phenotypic changes during liver fibrosis, we used recombinant CCL2 and its specific receptor antagonist. Moreover, we employed LX2 system to confirm our results.

Results: We found that hepatic M2 macrophages (CD163+) infiltration increased in different stages of liver fibrosis (F0-1: 34.95 \pm 18.12 ; F2-3: 77.57 \pm 32.48 ; F4: $99.62 \pm 40.84$, F0-1 vs. F2-3, P<0.001; F2-3 vs. F4, $\mathrm{P}=0.074$ ). After in vitro co-cultured with aHSCs, the macrophages expressed higher levels of CD163 ( $29.5 \pm 6.1 \%$ vs. $2.7 \pm 1.1 \%)$ as well as CD206 (28.0 $\pm 4.2 \%$ vs. $2.4 \pm 1.2 \%)$ compared with the control. Then we found aHSC supernatant up-regulated the expression of CD163 (26.1 $\pm 2.8 \%)$ and CD206 $(25.8 \pm 3.8 \%)$ on macrophages independently. We noted aHSCs' supernatant contained a high level of CCL2, which increased dramatically while TGF- $\beta$ stimulation. Meanwhile, CCL2 staining score increased with the

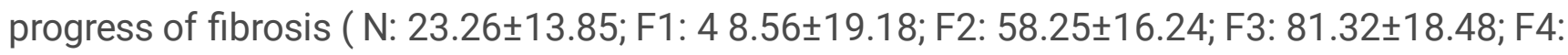
$110.93 \pm 24.75)$. Intriguingly, CCL2 significantly up-regulated the expression of CD163 $(27.6 \pm 7.0 \%)$ and CD206 (26.5 $\pm 5.1 \%)$ on macrophages besides inducing their aggregation. Results were confirmed with LX2 co-culture system.

Conclusions: (1) The expression of M2 macrophage marker CD163 increased significantly during the progress of liver fibrosis and associated with fibrosis severity. (2) AHSCs can recruit macrophages and induce their M2 phenotypic transformation through CCL2/CCR2 pathway.

\section{Background}

Liver cirrhosis - the final stage of liver fibrosis currently accounts for about 1.16 million deaths each year, ranking the 11th cause of death in the whole world [1]. The essence of fibrosis is the wound-healing response of chronic inflammation. It becomes problematic when dysregulated and excessive scarring occurs in response to persistent injury and leads to altered tissue functions [2, 3]. As liver is a particular immune organ, the immune system plays a dual role in the pathological process of fibrosis [3], which can 
mediate immune-inflammatory reactions and cause liver damage. The main pathological feature of cirrhosis is impaired liver immune system surveillance [4]. It has two extreme aspects: one is the systemic inflammatory response, which mainly due to incomplete and inappropriate activation of immune cells; the other is immunodeficiency, which is primarily caused by impaired response of the immune system to pathogens [4-6]. Some studies have shown that most immune cells, including monocytes/macrophages, NK cells, and lymphocytes, show impaired functions in varying degrees during cirrhosis $[5,7]$.

Macrophages featured for strong plasticity [8] can differentiate into both pro-fibrotic and anti-fibrotic macrophages responding to different conditions $[9,10]$. They could exert two opposed functions by produce pro-inflammation or pro-fibrotic factors in different circumstances [10-13]. Generally, classically activated macrophages (M1) usually secrete pro-inflammatory cytokines that exert anti-infective effects. And alternatively activated macrophages (M2) mainly regulate inflammation resolution and promote tissue repair[11,14]. The persistent inflammatory response in patients with chronic hepatitis suggests that the tolerance in liver immune cells might impede effective immune surveillance in the liver at certain situations $[5,6]$. It was reported that peripheral blood monocytes/macrophages are mainly M2 phenotype in the late stages of hepatitis B-related cirrhosis, which show decreased anti-infectivity, thereby increasing the possibility of bacterial infection [15]. In the clinical study, high M2-specific CD163 levels indicated poor prognosis and correlated with increased tumor nodules and venous infiltration in HCC patients [16]. However, the mechanisms about M2 macrophages formation during chronic liver diseases, especially liver fibrosis/cirrhosis, are not entirely clear.

During chronic liver injury, hepatic stellate cells resting in the sinus (the Disse) lumen receive signals from damaged hepatocytes, macrophages and other immune cells, and are rapidly activated into myofibroblast-like cells [17], also known as activated hepatic stellate cells (aHSCs) [18-21], which display fibrogenic properties mainly by producing excessive extracellular matrix and collagen [22]. Recently, more attention focus on the unexpected immune regulation roles of aHSCs in liver fibrosis [7, 21]. Our previous studies found that aHSCs can secrete a lot of immunomodulatory cytokines and chemokines to regulate the phenotype and function of monocytes, NK cells and T cells [23-25]. It is interesting to continue exploring and unveiling how aHSCs regulate the phenotype and function of macrophage in the long process of liver fibrosis.

CCL2, also known as monocyte chemotactic factor 1 (MCP1), mainly regulates migration and infiltration of monocytes/macrophages through combination with its specific receptor CCR2 [26, 27]. It has reported that CCL2/CCR2 axis plays a vital role during fibrosis [28-30]. CCL2-dependent infiltrating macrophages promote angiogenesis in progressive liver fibrosis and CCR2 is mainly responsible for the recruitment of proinflammatory and profibrogenic infiltrating monocytes during fibrosis progression [28]. Through the liquid chip screening, we found that CCL2 may profoundly enrich in the supernatant of aHSCs [23]. However, few studies explore if CCL2 independently affects the phenotype and function of monocytes/macrophages in the process of liver fibrosis. 
In this study, we found that aHSCs can aggregate macrophages and induce M2 phenotypic transformation through CCL2/CCR2 pathway during liver fibrosis.

\section{Methods}

\section{Patients and specimens}

Liver tissues with different stages of fibrosis were acquired from 96 patients undergoing curative liver resection for hepatocellular carcinoma who attended the Third Affiliated Hospital of Sun Yat-sen University in south China from 2014 to 2018. Patients with hepatitis C virus, HIV, Wilson's disease, autoimmune liver disease, genetic metabolic liver disease and other undefined pathogenesis liver diseases were excluded from our study. The fibrotic tissues were taken at least $3 \mathrm{~cm}$ away from the edge of the tumor according to other publications $[14,23,31]$. Fresh fibrotic liver tissues from 5 patients were selected for the acquisition of primary aHSCs. According to the Helsinki Declaration, all samples are anonymously coded in accordance with local ethical guidelines. The study was approved by the Institutional Review Board of the Third Affiliated Hospital of Sun Yat-sen University.

\section{Liver tissue immunohistochemistry and immunofluorescence staining}

The different stages of liver fibrosis were evaluated according to the Metavir score system with haematoxylin and eosin staining. Two independent observers blinded to the clinical outcome performed this analysis. Paraffin-embedded and formalin-fixed samples were processed for immunohistochemistry. After incubating with primary antibody targeted against human CD68, CD163 (Zsbio, China), and CCL2 (Sigma Aldrich, St. Louis, MO, USA), adjacent sections were stained with either diaminobenzidine or 3amino-9-ethylcarbazole using the Envision System (Dako Cytomation, Glostrup, Denmark). Five representative fields were selected at 20- magnification using microscopy (Leica, Mannheim, Germany). The M2 phenotype macrophages positive with CD163 staining were caculated per field. For the quantitation of CCL2 protein expression, the positive cells of each specimen was evaluated using Histoscore (H-score), calculated by a semi-quantitative assessment of both the intensity of staining (graded as: 0 , non-staining; 1 , weak; 2 , median; or 3 , strong) and the percentage of positive cells. The range of possible scores was from 0 to 300 . Expression level of each component was categorized as low or high according to the median value of the $\mathrm{H}$-score. Two independent pathologists blinded to the clinical outcome performed this analysis.

For immunofluorescence analysis, aHSCs were stained using rabbit anti-human alpha-smooth muscle actin (a-SMA, Abcam, Cambridge, MA, USA) and mouse anti-human CCL2 protein (Sigma Aldrich, St. Louis, MO, USA), followed by Alexa Fluor 555-conjugated donkey anti-mouse IgG and Alexa Fluor 488conjugated donkey anti-rabbit IgG (Invitrogen, Grand Island, NY, USA). Macrophages were stained using polyclonal mouse anti-human CD163 (DakoCytomation, Glostrup, Denmark). Positive cells were detected using immunofluorescence confocal microscopy (Leica, Germany). 


\section{In vitro co-culture system}

Primary aHSCs were isolated as described previously [31]. To minimize clonal selection and culture stress during extended culture, those between 3 to 8 passages were used for subsequent experiments. The LX2 cells (hepatic stellate cell line) were obtained from ATCC cell bank. THP-1 cells (kindly provided by Dr. Songguo Zheng), a human leukemia monocytic cell line, can be differentiated into macrophages under stimulation of $100 \mathrm{ng} / \mathrm{mL}$ 12-Otetradecanoylphorbol-I3-acetate (PMA) for 48 hours [32, 33]. The THP1derived MOMФ were cultured in RPMI 1640 containing 10\% FBS in 6-well flat-bottomed plates $\left(1 \times 10^{6}\right.$ cells/well) for at least 1 hour before they were ready for the co-culture with aHSCs (including primary aHSCs or TGF- $\beta$ activated LX2 at a ratio of 2:1 or 1:1). We used the LX2 cell line without any stimulation to mimic quiescent hepatic stellate cells as control. For the supernatant treatment group, we used $50 \%$ conditioned supernatant and $50 \%$ fresh complete medium to treat the macrophages for 72 hours before analysis. When indicated, recombinant human CCL2 protein ( $2 \mathrm{ng} / \mathrm{mL}, \mathrm{R} \& D$ Systems, Abingdon, UK) and INCB 3284 (100 ng/mL, Tocris Bioscience, UK) were added accordingly. At the indicated time intervals, the macrophages were harvested, counted and analyzed.

\section{Supernatants preparation and test}

Supernatants were generated by seeding $5 \times 10^{5}$ aHSCs or $1 \times 10^{6} \mathrm{LX} 2$ per well into 6 -well plates in $2 \mathrm{~mL}$ of DMEM with $10 \%$ Fetal Bovine Serum (FBS) containing $100 \mathrm{ng} / \mathrm{mL}$ benzylpenicillin and $100 \mathrm{ng} / \mathrm{mL}$ streptomycin (all purchased from Sigma Aldrich, St. Louis, MO, USA) for 2-3 days while more than $90 \%$ of cells confluence duly. The supernatants were harvested, centrifuged, and stored in aliquots at $-80^{\circ} \mathrm{C}$. The activated LX2 was prepared by stimulation with TGF- $\beta$ ( $2 \mathrm{ng} / \mathrm{mL}, \mathrm{R} \& D$ Systems, Abingdon, UK) for no less than 24 hours to ensure the complete activation, and then replaced the medum with fresh medium without TGF- $\beta$ for another 24 hours before harvesting the supernatant. CCL2 levels of the supernatants were tested with CCL2 enzyme-linked immunosorbent assays (ELISA, R\&D Systems, Abingdon, UK) according to the instructions.

\section{Flow cytometry and real-time quantitative polymerase chain reaction (qPCR)}

Monocytes/macrophages $\left(1 \times 10^{6}\right)$ were stained with fluorochrome-conjugated monoclonal antibodies of CD14, CD163 and CD206 (all purchased from BD Biosciences) according to the manufacturer's instructions and then analyzed by flow cytometry. Data were acquired and analyzed on the Flow Cytometer (BD Biosciences). Total RNA was purified from cultured macrophages using TriPure Isolation Reagent Kit (Roche Applied Science, Germany). cDNA synthesis was undertaken using the Transcriptor first-strand cDNA synthesis kit (Roche Diagnostics, Mannheim, Germany). Quantitative RT-PCR was performed with the SYBR Green PCR kit (Roche Diagnostics, Mannheim, Germany) using the LightCycler 480 apparatus (Roche, Mannheim, Germany). Relative concentrations were calculated and normalized with housekeeping gene $\beta$-actin as control. Primers were used to amplify the following mRNAs: CCR2, 
CD163, ARG1, IL-10, according to the standard protocol. Primer sequences were provided in the supplementary materials.

\section{Chemotaxis experiments}

In the standard assay, the upper chamber was seeded with macrophages and was separated by a porous insert from a lower chamber, into which the cells would migrate. In the lower chamber, aHSCs were seeded with or without CCR2 antagonist INCB 3284. At the desired time, macrophages that migrated to the underside of the insert could be fixed and stained for quantitation by microscopy.

\section{Statistical analysis}

Statistical analysis was performed using the SPSS 22.0. Data conforming to the normal distribution were expressed as mean \pm standard deviation, using independent sample t-test, paired t-test. Non-normally distributed data were compared by the Mann-Whitney $U$ test, two-tailed test. P values inferior to 0.05 were considered significant difference.

\section{Results}

\section{M2 macrophages $\left(\mathrm{CD}_{163^{+}}\right)$infiltration increased as the progress of liver fibrosis.}

To examine the relationship between hepatic M2 macrophages and liver fibrosis stages, we first compared the expression of CD163 which is mainly expressed on M2 macrophages in different stages of hepatic fibrosis among patients. The characteristics of the patients involved in our study were shown in Table 1. The degree of liver fibrosis in different patients was assessed through hematoxylin-eosin staining according to the Metavir score system, as shown in Figure 1A. F0-1, F2-3 and F4 fibrosis status were defined as mild, moderate and severe degree, respectively. As shown in Figure 1B and $\mathrm{C}$, the expression of CD163 in liver tissues increased significantly as the fibrosis aggravated, with the IHC score $34.95 \pm 18.12$ in mild degree, $77.57 \pm 32.48$ in moderate degree and $99.62 \pm 40.84$ in severe degree (mild vs. moderate, $\mathrm{P}<0.001$; moderate vs. severe, $\mathrm{P}=0.007$ ). These results indicated that $\mathrm{M} 2$ macrophages increased obviously during the progress of fibrosis. We prospected that the fibrosis environment may affect the phenotype of macrophages.

\section{MOMФ developed into M2 phenotype when co-cultured with aHSCs or treated with supernatant from aHSCs}

Since hepatic stellate cells are always activated during liver fibrosis and can secret many kinds of cytokines and chemokines [23], we wondered if the aHSCs were responsible for monocytes infiltration and $\mathrm{M} 2$ phenotype formation in fibrotic livers. We successfully extracted five cases of primary human aHSCs with sustainable and stable growth. To investigate the regulation of aHSCs on macrophages, the THP-1-derived MOMФ were co-cultured with primary aHSCs at a ratio of 5:1. After 5 days of co-culture, the expression of CD163 and CD206 on macrophages were detected. Compared with the control group, the macrophages co-cultured with aHSCs expressed high levels of M2 phenotype-specific proteins: CD163 
$(29.5 \pm 6.1 \%$ vs $2.7 \pm 1.1 \%, P<0.001)$ and CD206 (28.0 $\pm 4.2 \%$ vs $2.4 \pm 1.2 \%, P<0.001)$, as shown in Figure 2. The results indicate that aHSCs have a significant regulatory effect on macrophages through certain pathways, which might promote the M2 phenotype differentiation.

To further explore the mechanism of aHSCs' immunomodulatory effects on macrophages and to evaluate the cell-cell interaction effect during the co-culture system, we detected MOMФ phenotypic changes under the treatment of aHSCs supernatant. As shown in Figure 2, the supernatant treated group could independently up-regulate the expression of CD163 and CD206 on macrophages compared with the control group ( $26.1 \pm 2.8 \%$ vs. $2.7 \pm 1.1 \%, P<0.001$ and $25.8 \pm 3.8 \%$ vs. $2.4 \pm 1.2 \%, P<0.001)$, indicating that the aHSCs may secrete specific cytokines responsible for the macrophages' phenotype transformation. Of note, macrophages in the supernatant group showed relatively lower expression of CD163 and CD206 compared with the co-culture group, indicating that cell to cell contact may help to induce other ways to promote M2 phenotype transformation besides soluble molecules.

\section{AHSCs secret high levels of CCL2}

In our previous study, we found that aHSCs secreted a variety of cytokines and chemokines including CCL2 [23]. It has acknowledged that CCL2 can recruit immune cells including monocytes, and we wondered if CCL2 might also play a role in upregulating the expression of CD163 and CD206 on macrophages. Firstly, we would like to further confirm the production of CCL2 in aHSCs as well as in those with much stronger activated status under the stimulation of TGF- $\beta$. To better compare the results and get much more robust data, we used LX2 cell lines as another control. As shown in Figure 3A, the primary aHSCs are typically fusiform, and express the activation marker a-SMA, together with a high expression of CCL2 protein. The production of CCL2 in the supernatant of the primary aHSC, TGF- $\beta$ (2 $\mathrm{ng} / \mathrm{mL})$ stimulated aHSCs, LX2 and TGF- $\beta(2 \mathrm{ng} / \mathrm{mL})$ activated LX2 were tested by ELISA. As shown in Figure 3B, aHSCs can secrete high levels of CCL2 compared with LX2, and interestingly this ability can be further enhanced with stronger activation induced by TGF- $\beta$ (LX2 vs. TGF- $\beta$ stimulated LX2, $P<0.001$; aHSC vs. TGF- $\beta$ stimulated aHSCs, $P<0.001)$.

Secondly, consistent with the in vitro study, we found that the expression of hepatic CCL2 increased as the progress of liver fibrosis among the patients. As shown in Figure 3C, as the degree of liver fibrosis worsen, the expression of CCL2 gradually increased compared to the healthy control group (CCL2 staining score of $\mathrm{N}$ is $23.26 \pm 13.85 ; \mathrm{F} 1: 48.56 \pm 19.18, \mathrm{P}=0.03$; F2: $58.25 \pm 16.24, \mathrm{P}<0.001$; F3: $81.33 \pm$ $18.47, P<0.001 ; F 4: 110.93 \pm 24.75, P<0.001)$. Of note, besides the significantly increase in both marker (CCL2 and CD163) as the progress of fibrosis (Figure 3D), there was a relatively strong relationship between M2 macrophage (CD163+) IHC score and CCL2 IHC score $(r=0.40, P<0.05)$ further strengthening our hypothesis that CCL2 may regulate M2 phenotype transformation.

\section{AHSCs induce macrophage infiltration and M2 differentiation via CCL2}

To verify that CCL2 is indeed responsible for macrophage infiltration and differentiation into the M2 phenotype during liver fibrosis, we used recombinant human CCL2 (Rh CCL2) and its receptor antagonist 
INCB to confirm this pathway further. As shown in Figure 4A-B, macrophage infiltration increased significantly when aHSCs were cultured in the lower chamber compared to control (only medium), and the number decreased while we used CCL2 specific receptor antagonist INCB to block CCL2/CCR2 pathway in the same culture system. Rh CCL2 could also mimic aHSCs on the ability of macrophage infiltration, indicating that aHSCs may recruit macrophage infiltration mainly through CCL2.

As what we prospected, CD163 and CD206 expression significantly up-regulated on macrophages under the stimulation of Rh CCL2 compared to the control group as shown in Figure 4C-D (CD163: $27.6 \pm 7.0 \%$ vs $2.7 \pm 1.1 \%, P=0.008 ; C D 206: 26.5 \pm 5.1 \%$ vs $2.4 \pm 1.2 \%, P=0.003)$. The addition of INCB $(100 \mathrm{ng} / \mathrm{mL})$ prohibited the expression of CD163 and CD206 on the MOMФ (CD163: $4.5 \pm 1.4 \%$, CD206: $4.1 \pm 2.6 \%$, vs MO NC group, $P>0.05$ ). To avoid the clonal selection and individual differences of the primary aHSCs, we used LX2 cell lines to repeat the experiment. As shown in Figure 5, LX2 activated under the stimulation of TGF- $\beta$ could upregulate the expression of CD163 and CD206 on macrophages both in the co-culture system and in supernatant stimulation way. Considering activated LX2 also produced large amounts of CCL2 (Figure 3B) and CCL2 specific receptor antagonist INCB could block their modulation function of macrophages (Figure $4 \mathrm{C}$ and $\mathrm{D}$ ), we concluded that aHSCs indeed induce macrophage M2 phenotype differentiation through CCL2/CCR2 pathway.

\section{AHSCs upregulate macrophage CCR2 expression to enhance the function of CCL2/CCR2 pathway}

Interestingly, while we used qPCR to test M2MФ specific markers expression in mRNA levels after treating THP-1 derived MOMФ at different conditions (supernatants from aHSC, aLX2, LX2) for 5 days, we found that macrophage CCR2 expression was upregulated besides CD163, ARG-1, and IL-10 (Figure 6A). We further confirmed the upregulation of both CD163 and CCR2 on Rh CCL2 stimulated macrophages by immunofluorescence and APCR (Figure 6B-C). However, while we used only Rh CCL2 $(5 \mathrm{ng} / \mathrm{mL})$ to treat macrophages, the expression of CCR2 seemed lower than that of treated by aHSCs supernatant (aHSCs supernatant group vs. control, $\mathrm{P}=0.001$; Rh CCL2 group vs. control, $\mathrm{P}=0.008$; aHSCs supernatant vs. $\mathrm{Rh}$ $\mathrm{CCL} 2, \mathrm{P}=0.169$ ). Other mechanisms should be there to increase the expression of CCR2 besides CCL2. In conclusion, these results indicated that besides the secretion of high levels of CCL2, aHSCs could also upregulate the expression of CCR2 on macrophages to activate the CCL2/CCR2 pathway. CCL2 can independently activate this pathway since Rh CCL2 upregulated macrophage CCR2 expression in our experiment, while other factors produced from aHSCs may strengthen this pathway as well.

\section{Disscussion}

This study focused on the immunoregulatory function of aHSCs during liver fibrosis, primarily explored their roles in the regulation of macrophage phenotype and function. We showed that M2 macrophages $\left(C D 163^{+}\right)$infiltration increased significantly as the progress of liver fibrosis, accompanied by the upregulation of CCL2 expression in the fibrotic liver tissues. We demonstrated in vitro that primary aHSCs isolated from fibrotic liver tissues could produce high level of CCL2, promoting the conversion of macrophages to the M2 phenotype (high expression of CD163), which would display their 
immunosuppressive functions by secreting inhibitory cytokines such as IL-10, ARG1, etc. This may contribute to the immunosuppressive status in the liver and afford somewhat clues for the explanations of increased risk of hepatocellular carcinoma and bacterial infection in patients with cirrhosis [16, 34].

CD163 is a multifunctional receptor involved in receptor-mediated endocytosis and signal pathways upon interaction with diverse ligands. CD163-positive macrophages are usually found during the healing phase of acute inflammation and chronic inflammation in wound-healing tissues, whereas freshly infiltrated macrophages are CD163-negative [35]. CD163 is suggested to act as an innate immune sensor for bacteria and inducer of local immunity, rather than as a phagocytic receptor and has been proposed as an anti-inflammatory marker for macrophages [36]. CD163 is shed from the macrophage surface into the circulation upon activation of cell surface Toll-like receptors (TLRs) and is found in the blood as soluble CD163 (sCD163). It is reported that SCD163 levels are increased in patients with chronic viral hepatitis and increased SCD163 is associated with the severity of disease and predicts fibrosis [37-39]. It is also determined that the SCD163 serum level is a new independent noninvasive risk factor for death and variceal bleeding in cirrhotic patients [40]. However, the link between hepatic CD $163^{+}$macrophages accumulation and liver fibrosis progression remain elusive, and experimental evidence for the reasons why CD163 upregulation during liver fibrosis is still lacking. We found that the expression of CD163 in liver tissues increased significantly as the fibrosis aggravated. This may explain the reason for the increase of SCD163 levels in patients' serum since SCD163 is shed from activated macrophages which mainly locate in the liver.

The next important issue is how the macrophages express high level of CD163 during liver fibrosis. Previous studies suggested that elevated CCL2 expression is mainly associated with the monocytes/macrophages infiltration in liver injury and acts as a critical component of inflammatory damage [41-43]. Interestingly, we found that CCL2 improve THP1-derived MOMФ differentiation into $\mathrm{CD}_{163^{+}}$macrophages independently. We speculated that, besides the recruitment of immune cells, CCL2 might also play immune modulation roles which were underestimated before. Indeed, it showed that the supernatant from aHSCs upregulated the expression of CD163 on macrophages much more obviously than CCL2 itself, which indicated that other factors in aHSCs supernatant might also induce the expression of CD163. It is reported that the expression of CD163 could be induced by glucocorticoids, IL10, IL-6 and M-CSF [35]. Actually, we found aHSCs can secrete high levels of IL-6 and M-CSF in our previous study [23], which may explain why aHSCs showed stronger upregulation function than Rh CCL2 alone and also indicated complicated immune modulation pathways during liver fibrosis where aHSCs dominate.

Recent studies showed that the CCL2/CCR2 axis plays a vital role in the fibrotic formation in some diseases, including pulmonary fibrosis, renal fibrosis and nonalcoholic liver fibrosis, in addition to chemotactic macrophage infiltration $[27,44,45]$. It is found that CCL2/CCR2 axis was closely related to aggregation of myeloid-derived inhibitory cells (MDSCs) and inhibition of T cell function in pulmonary fibrosis and lung cancer [46]. The proportions of $\mathrm{CD} 11 \mathrm{C}^{+} \mathrm{CD} 206^{+}$and $\mathrm{CCR} 2^{+}$macrophages in adipose tissues highly elevated in patients with NASH compared to healthy controls and to patients with fatty liver 
and CCR2 ${ }^{+}$macrophages were also correlated with NASH severity (44). It seems that CCR2 ${ }^{+}$ macrophages play essential roles in inflammation, cancer, fibrosis and etc. However, few studies ask the reason why and how CCR2 be upregulated on macrophages. Our study revealed the correlation between high expression of CCL2, macrophage infiltration and the hepatic fibrosis progress, and found that CCL2 increased strikingly with the continuous activation of hepatic stellate cells, which is the central part of cirrhosis. And more importantly, we found that CCL2 produced by aHSCs can independently upregulate CCR2 expression on macrophages, and some other factors from aHSCs may also help to stimulate CCR2 expression. This may provide somewhat clues and explanations to the CCR $2^{+}$macrophages recruitment most other studies have omited.

By cell culture systems, we proved aHSCs could induce macrophages aggregation and M2 phenotype differentiation. This effect was strengthened as the hepatic stellate cells further activated and disappeared when the CCL2/CCR2 axis was inhibited by INCB. On the one hand, M2 macrophage relieve liver damage caused by inflammatory reactions, and on the other hand, they accelerate the immune surveillance disorder under cirrhotic condition by secreting immunosuppressive cytokines [4], promoting vascular regeneration and tissue reconstruction [12]. What's more, M2 macrophages show a relatively weak antigen presentation, and secrete IL-10, TGF- $\beta$ and other cytokines which are beneficial to the survival and sustained activation of aHSCs [34]. Therefore, we propose that during the progression of liver fibrosis (especially HBV related), there might exist the "amplification loop" between aHSCs and macrophages, and CCL2/CCR2 axis plays essential roles in this loop. Since there was a study found some antifibrotic effects of CCL2 inhibitor in animal models of liver fibrosis [30], it is worth to continue to explore the pathway of CCL2/CCR2 as potential therapeutic targets in the future.

We also acknowledge our study limitations. Firstly, due to the limited experiment conditions, we can't confirm our results in animal models especially transgenic mice models to investigate the detailed mechanisms about how CCL2 produced by aHSCs affects the function of macrophages and the whole immune status during live fibrosis. Secondly, we can't get the conclusion about what is the main function of the upregulation of CD163 and CCR2 on macrophages during liver fibrosis. Although some experiments about CD163 and CCR2 + macrophages gave us hints that this kind of macrophages might contribute to immune suppression status in the liver and might help the development of HCC and bacterium infection [34], we can't show some robust data and direct evidences to prove these hypotheses. Thirdly, all our in vitro experiments were based on the THP-1 cells, a human leukemia monocytic cell line. We are not sure if this reflects the same situation in that of the primary normal human monocytes. It is warranted to repeat all the experiments with primary human monocytes if possible.

\section{Conclusion}

In conclusion, our study suggests that patients with HBV related fibrosis showed increased expression of hepatic CD163 and CCL2, and their expression increased dramatically as the progress of liver fibrosis. There might be an "amplification loop" between aHSCs and macrophages through CCL2/CCR2 axis, and more studies on this area are worth to try both in vitro and in vivo. 


\section{Abbreviations}

aHSCs

activated hepatic stellate cells;

CCL2

C-C motif chemokine ligand 2;

CCR2

C-C Motif Chemokine Receptor 2;

МОМФ

THP-1-derived M0-type macrophages;

MCP1

monocyte chemotactic factor 1 ;

HCC

hepatocellular carcinoma;

HIV

human immunodeficiency virus;

$\mathrm{H}$-score

Histoscore;

PMA

12-Otetradecanoylphorbol-I3-acetate;

FBS

Fetal Bovine Serum;

qPCR

quantitative polymerase chain reaction;

ELISA

enzyme-linked immunosorbent assays;

TLRs

Toll-like receptors;

MDSCs

myeloid-derived inhibitory cells;

$\mathrm{NASH}$

non-alcoholic steatohepatitis.

\section{Declarations}

\section{Ethics declarations}

\section{Ethics approval and consent to participate}

Human subjects: The study was approved by the Institutional Review Board of the Third Affiliated Hospital of Sun Yat-sen University. 
Animals: Not applicable.

\section{Consent for publication}

All authors approved the manuscript for the publication.

\section{Competing interests}

The author(s) indicated no potential conflicts of interest.

\section{Corresponding authors}

Correspondence to Yutian Chong or Min Zhang.

\section{Funding}

This work was supported by grants from the Science and Technology Planning Projects of Guangzhou City (201707010005, 201804010474), Guangdong Natural Science Foundation (2016A030313250》 2017A030313711), Natural Science Foundation (81773176), Sun Yat - sen University Clinical Medicine Research 5010 Project (2016009).

\section{Authors' contributions}

YC, MZ conceived, designed the study. SX and XZ collected the data, performed the research, and wrote the manuscript, $S X$ and $Y J$ performed the cell isolation, YW, JG, YJ, XL performed the data analysis, JC and LS collected the data, MZ, YJ and YC revised the manuscript.

\section{Acknowledgements}

Not applicable.

\section{Availability of data and material}

Supplementary Table 1 Primers used during the study.

\section{References}

1. Asrani SK, Devarbhavi H, Eaton J, Kamath PS. Burden of liver diseases in the world. J Hepatol. 2019;70:151-71.

2. Friedman SL. Mechanisms of hepatic fibrogenesis. Gastroenterology. 2008;134:1655-69.

3. Pellicoro A, Ramachandran P, Iredale JP, Fallowfield JA. Liver fibrosis and repair: immune regulation of wound healing in a solid organ. Nat Rev Immunol. 2014;14:181-94. 
4. Wilde B, Katsounas A. Immune Dysfunction and Albumin-Related Immunity in Liver Cirrhosis. Mediators of Inflammation 2019:9.

5. Dirchwolf M, Podhorzer A, Marino M, Shulman C, Cartier M, Zunino M, et al. Immune dysfunction in cirrhosis: Distinct cytokines phenotypes according to cirrhosis severity. Cytokine. 2016;77:14-25.

6. Albillos A, Lario M, Alvarez-Mon M. Cirrhosis-associated immune dysfunction: Distinctive features and clinical relevance. J Hepatol. 2014;61:1385-96.

7. Mehrfeld C, Zenner S, Kornek M, Lukacs-Kornek V. The Contribution of Non-Professional AntigenPresenting Cells to immunity and Tolerance in the Liver. Frontiers in Immunology 2018, 9:8.

8. Wynn TA, Chawla A, Pollard JW. Macrophage biology in development, homeostasis and disease. Nature. 2013;496:445-55.

9. Porcheray F, Viaud S, Rimaniol AC, Leone C, Samah B, Dereuddre-Bosquet N, et al. Macrophage activation switching: an asset for the resolution of inflammation. Clin Exp Immunol. 2005;142:4819.

10. Tacke F, Zimmermann HW. Macrophage heterogeneity in liver injury and fibrosis. J Hepatol. 2014;60:1090-6.

11. Muliaditan T, Caron J, Okesola M, Opzoomer JW, Kosti P, Georgouli M, et al: Macrophages are exploited from an innate wound healing response to facilitate cancer metastasis. 2018, 9:2951.

12. Faure-Dupuy S, Durantel D, Lucifora J. Liver macrophages: Friend or foe during hepatitis B infection? Liver International 2018, 38:1718-1729.

13. Duffield JS, Forbes SJ, Constandinou CM, Clay S, Partolina M, Vuthoori S, et al. Selective depletion of macrophages reveals distinct, opposing roles during liver injury and repair. Journal of Clinical Investigation. 2005;115:56-65.

14. Kuang DM, Zhao Q, Peng C, Xu J, Zhang JP, Wu C, et al. Activated monocytes in peritumoral stroma of hepatocellular carcinoma foster immune privilege and disease progression through PD-L1. J Exp Med. 2009;206:1327-37.

15. Antoniades CG, Berry PA, Davies ET, Hussain M, Bernal W, Vergani D, et al. Reduced monocyte HLADR expression: a novel biomarker of disease severity and outcome in acetaminophen-induced acute liver failure. Hepatology. 2006;44:34-43.

16. Yeung OW, Lo CM, Ling CC, Qi X, Geng W, Li CX, et al. Alternatively activated (M2) macrophages promote tumour growth and invasiveness in hepatocellular carcinoma. J Hepatol. 2015;62:607-16.

17. Wynn TA. Cellular and molecular mechanisms of fibrosis. J Pathol. 2008;214:199-210.

18. Tsochatzis EA, Bosch J, Burroughs AK. Liver cirrhosis. Lancet. 2014;383:1749-61.

19. Seki E, Schwabe RF. Hepatic inflammation and fibrosis: functional links and key pathways. Hepatology. 2015;61:1066-79.

20. Lee YA, Wallace MC, Friedman SL. Pathobiology of liver fibrosis: a translational success story. Gut $2015,64: 830-841$. 
21. Seki E, Brenner DA. Recent advancement of molecular mechanisms of liver fibrosis. J Hepato-BiliaryPancreat Sci. 2015;22:512-8.

22. Tsuchida T, Friedman SL. Mechanisms of hepatic stellate cell activation. Nature Reviews Gastroenterology Hepatology. 2017;14:397-411.

23. Zhang M, Wang FL, Zhu JY, Zheng YB, Zhao QY, Gu YR, et al. Liver myofibroblasts regulate the phenotype and function of monocytes through soluble factors in cirrhosis. Exp Ther Med. 2013;5:143-9.

24. Zhang M, Ye Y, Wang F, Zhu J, Zhao Q, Zheng Y, et al. Liver myofibroblasts up-regulate monocyte CD163 expression via PGE2 during hepatitis B induced liver failure. J Transl Med. 2014;12:60.

25. Zhang M, Wang F, Chong Y, Tai Q, Zhao Q, Zheng Y, et al. Liver myofibroblasts from hepatitis $B$ related liver failure patients may regulate natural killer cell function via PGE2. J TransI Med. 2014;12:308.

26. Deshmane SL, Kremlev S, Amini S, Sawaya BE. Monocyte Chemoattractant Protein-1 (MCP-1): An Overview. J Interferon Cytokine Res. 2009;29:313-26.

27. Osafo-Addo AD, Herzog EL. CCL2 and T cells in pulmonary fibrosis: an old player gets a new role. Thorax. 2017;72:967-8.

28. Ehling J, Bartneck M, Wei X, Gremse F, Fech V, Mockel D, et al: CCL2-dependent infiltrating macrophages promote angiogenesis in progressive liver fibrosis. Gut 2014, 63:1960-1971.

29. Karlmark KR, Weiskirchen R, Zimmermann HW, Gassler N, Ginhoux F, Weber C, et al. Hepatic recruitment of the inflammatory $\mathrm{Gr} 1+$ monocyte subset upon liver injury promotes hepatic fibrosis. Hepatology. 2009;50:261-74.

30. Baeck C, Wei X, Bartneck M, Fech V, Heymann F, Gassler N, et al. Pharmacological inhibition of the chemokine $\mathrm{C}-\mathrm{C}$ motif chemokine ligand 2 (monocyte chemoattractant protein 1 ) accelerates liver fibrosis regression by suppressing $\mathrm{Ly}-6 \mathrm{C}(+)$ macrophage infiltration in mice. Hepatology. 2014;59:1060-72.

31. Li XY, Su YJ, Hua XF, Xie C, Liu J, Huang YH, et al: Levels of hepatic Th17 cells and regulatory T cells upregulated by hepatic stellate cells in advanced HBV-related liver fibrosis. Journal of Translational Medicine 2017, 15:11.

32. Chanput W, Mes JJ, Wichers HJ. THP-1 cell line: An in vitro cell model for immune modulation approach. Int Immunopharmacol. 2014;23:37-45.

33. Cousins RJ, Blanchard RK, Popp MP, Liu L, Cao J, Moore JB, et al. A global view of the selectivity of zinc deprivation and excess on genes expressed in human THP-1 mononuclear cells. Proc Natl Acad Sci USA. 2003;100:6952-7.

34. Tacke F. Targeting hepatic macrophages to treat liver diseases. J Hepatol. 2017;66:1300-12.

35. Van Gorp H, Delputte PL, Nauwynck HJ. Scavenger receptor CD163, a Jack-of-all-trades and potential target for cell-directed therapy. Mol Immunol. 2010;47:1650-60. 
36. Fabriek BO, van Bruggen R, Deng DM, Ligtenberg AJ, Nazmi K, Schornagel K, et al. The macrophage scavenger receptor CD163 functions as an innate immune sensor for bacteria. Blood. 2009;113:88792.

37. Laursen TL, Wong GL, Kazankov K, Sandahl T, Moller HJ, Hamilton-Dutoit S, et al. Soluble CD163 and mannose receptor associate with chronic hepatitis $\mathrm{B}$ activity and fibrosis and decline with treatment. J Gastroenterol Hepatol. 2018;33:484-91.

38. Kuniholm MH, Hanna DB, Landay AL, Kaplan RC, Ley K. Soluble CD163 is associated with noninvasive measures of liver fibrosis in hepatitis $C$ virus- and hepatitis $C$ virus/human immunodeficiency virus-infected women. Hepatology 2015, 61:734-735.

39. Kazankov K, Barrera F, Moller HJ, Bibby BM, Vilstrup H, George J, et al: Soluble CD163, a Macrophage Activation Marker, Is Independently Associated With Fibrosis in Patients With Chronic Viral Hepatitis B and C. Hepatology 2014, 60:521-530.

40. Waidmann O, Brunner F, Herrmann E, Zeuzem S, Piiper A, Kronenberger B. Macrophage activation is a prognostic parameter for variceal bleeding and overall survival in patients with liver cirrhosis. $J$ Hepatol. 2013;58:956-61.

41. Mitchell C, Couton D, Couty JP, Anson M, Crain AM, Bizet V, et al. Dual role of CCR2 in the constitution and the resolution of liver fibrosis in mice. Am J Pathol. 2009;174:1766-75.

42. Ambade A, Lowe P, Kodys K, Catalano D, Gyongyosi B, Cho Y, et al. Pharmacological Inhibition of CCR2/5 Signaling Prevents and Reverses Alcohol-Induced Liver Damage, Steatosis, and Inflammation in Mice. Hepatology. 2019;69:1105-21.

43. Boniakowski AE, Kimball AS, Joshi A, Schaller M, Davis FM, denDekker A, et al. Murine macrophage chemokine receptor CCR2 plays a crucial role in macrophage recruitment and regulated inflammation in wound healing. Eur J Immunol. 2018;48:1445-55.

44. Gracia-Sancho J, Marrone G, Fernandez-Iglesias A: Hepatic microcirculation and mechanisms of portal hypertension. Nature reviews Gastroenterology \& hepatology 2018.

45. Miura K, Yang L, van Rooijen N, Ohnishi H, Seki E. Hepatic recruitment of macrophages promotes nonalcoholic steatohepatitis through CCR2. Am J Physiol Gastrointest Liver Physiol. 2012;302:G1310-21.

46. Wang Y, Zhang X, Yang L, Xue J, Hu G. Blockade of CCL2 enhances immunotherapeutic effect of anti-PD1 in lung cancer. J Bone Oncol. 2018;11:27-32.

\section{Tables}




\begin{tabular}{|c|c|c|}
\hline Demographic or Characteristics & Number & $\%$ \\
\hline \multicolumn{3}{|l|}{ Gender } \\
\hline Female & 15 & 15.63 \\
\hline Male & 81 & 84.37 \\
\hline \multicolumn{3}{|l|}{ Age } \\
\hline$\leq 40$ & 15 & 15.63 \\
\hline $41-50$ & 22 & 22.92 \\
\hline $51-60$ & 36 & 37.5 \\
\hline $61-70$ & 19 & 19.79 \\
\hline$>70$ & 4 & 4.16 \\
\hline \multicolumn{3}{|l|}{ Fibrosis } \\
\hline F0-1(Mild) & 21 & 21.88 \\
\hline F2-3 (Moderate) & 34 & 35.42 \\
\hline F4(Severe/cirrhosis) & 41 & 42.70 \\
\hline
\end{tabular}

Figures 
A

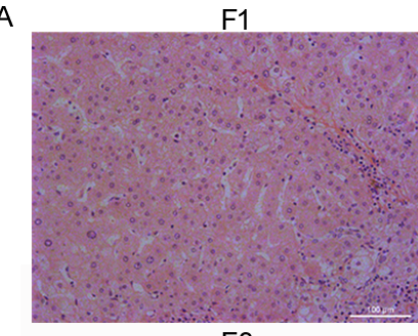

F3

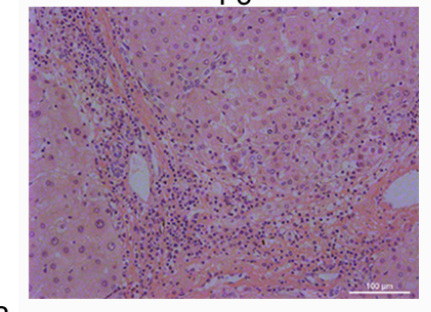

$\mathrm{F} 1$

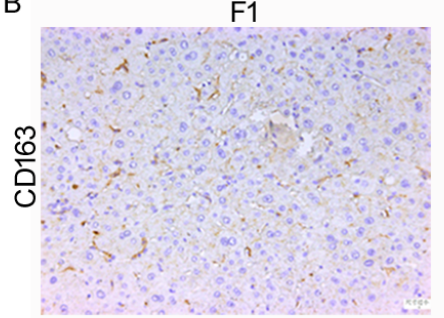

F3

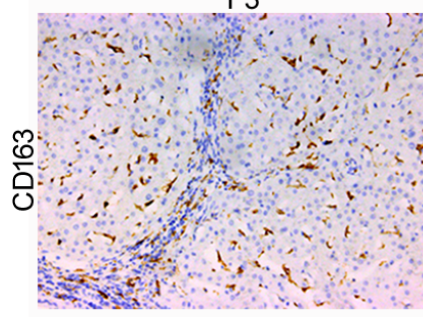

F2

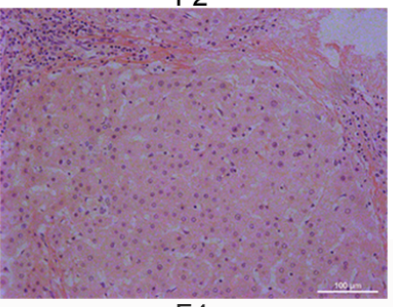

F4

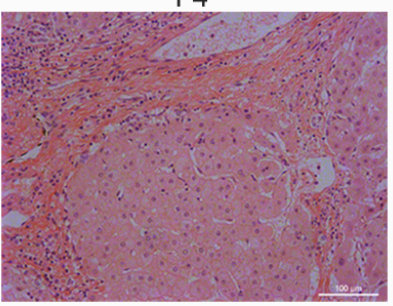

F2

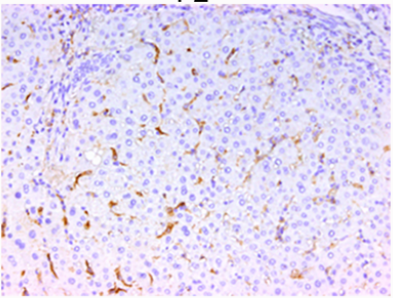

F4

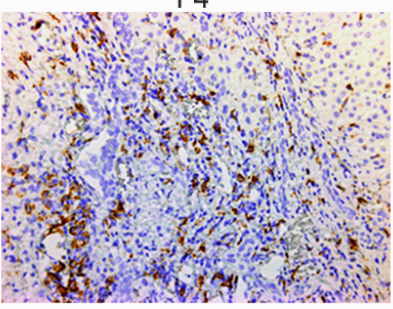

C

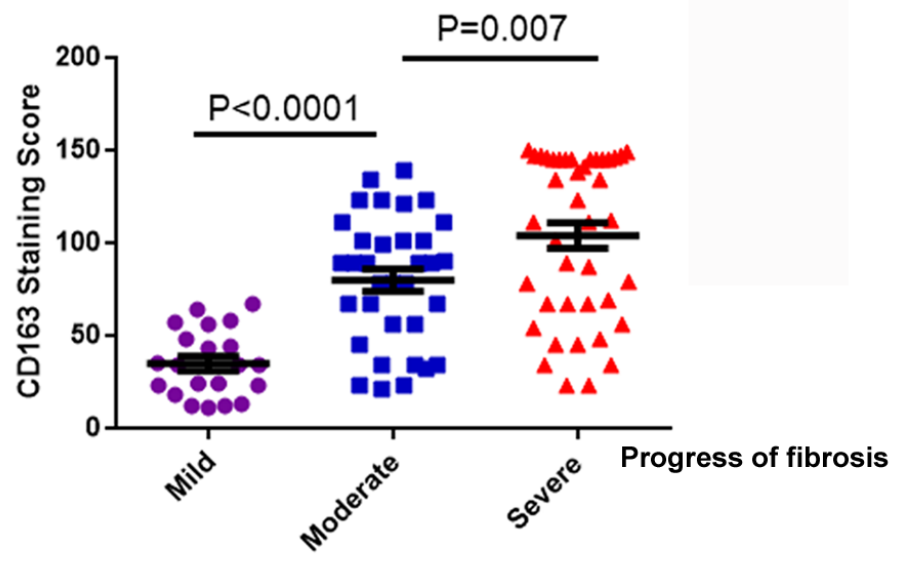

Figure 1

M2 macrophages (CD163+) infiltration increased as the progress of liver fibrosis. A. F1, fibrosis around the portal vein; F2, fibrous interval around the portal vein; F3, a large number of fibrous intervals are formed between the portal vein and the central vein; F4, cirrhosis. HE staining, the grade of liver fibrosis was based on Metavir score, $\times 200$ times. B. The expression of CD163 in liver tissues increased significantly as the fibrosis aggravated. F0-1, F2-3, F4 were respectively defined as the mild, moderate, 
severe degree. The CD163 IHC score in the mild degree was $34.95 \pm 18.12$, moderate was $77.57 \pm 32.48$, severe was $99.62 \pm 40.84$, and mild vs. moderate, $P<0.001$, moderate vs. severe, $P=0.007$.

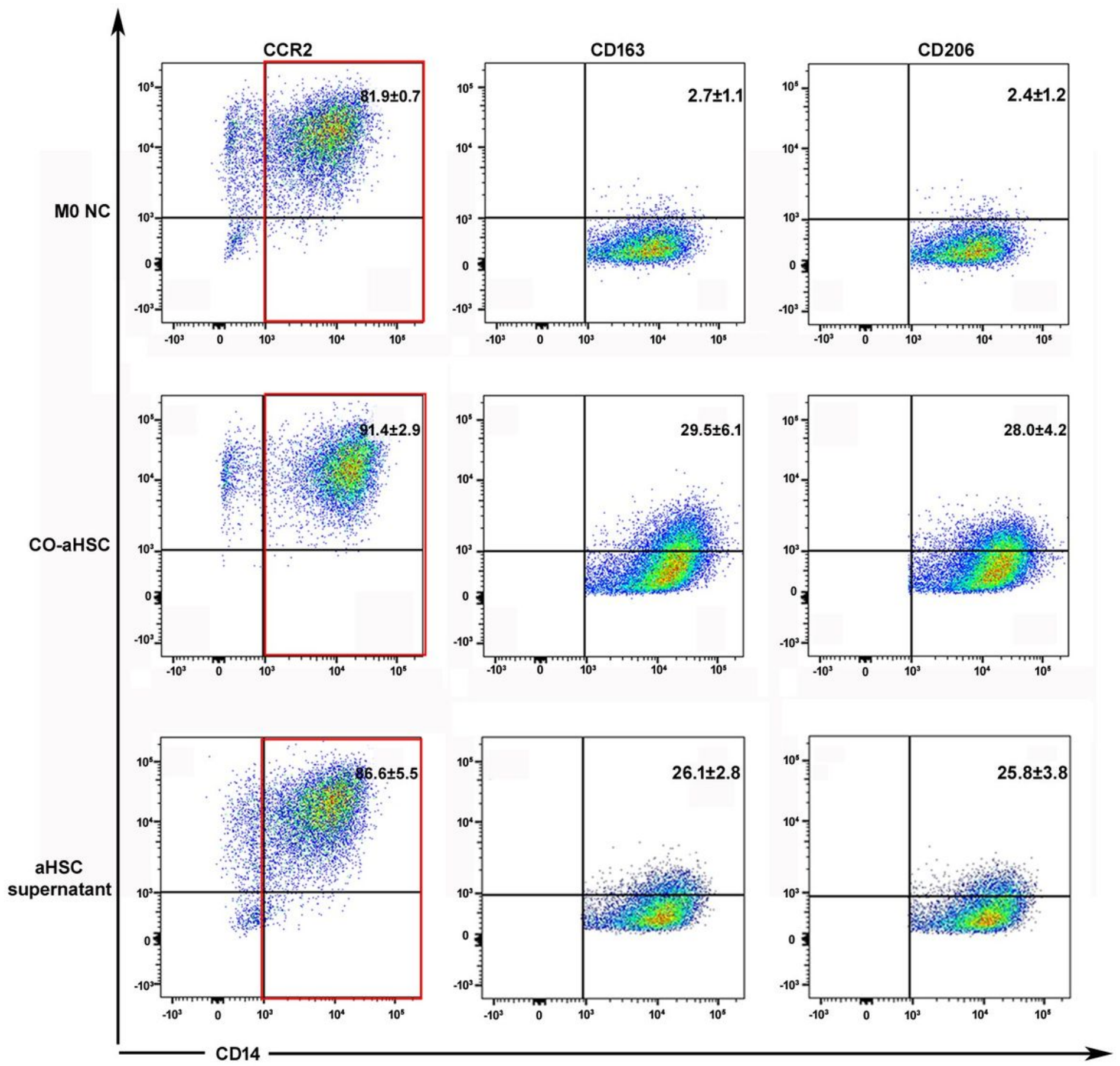

Figure 2

Either the co-culture condition with aHSCs or their supernatant could independently induce M2 macrophage differentiation. After 5.5 days, compared with the control group (MO NC), the co-aHSC group highly expressed M2 phenotype specific proteins: CD163 (29.5 $\pm 6.1 \%$ vs $2.7 \pm 1.1 \%, P<0.001)$ and CD206 $(28.0 \pm 4.2 \%$ vs $2.4 \pm 1.2 \%, P<0.001)$, the aHSC supernatant group could independently up-regulate the 
expression of CD163 and CD206 on macrophages compared with the M0 NC group $(26.1 \pm 2.8 \%$ vs $2.7 \pm$ $1.1 \%, P<0.001$ and $25.8 \pm 3.8 \%$ vs $2.4 \pm 1.2 \%, P<0.001)$.

A

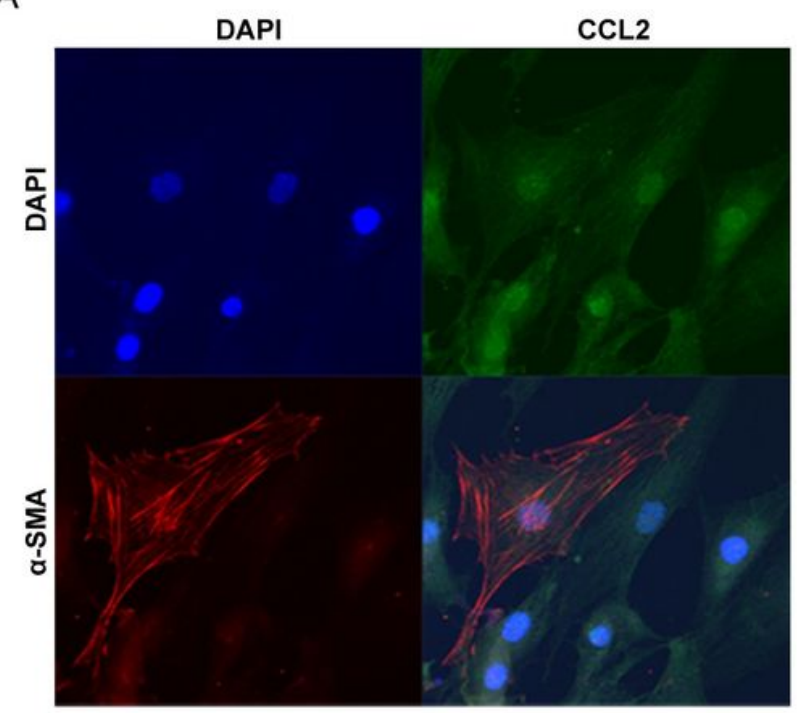

B

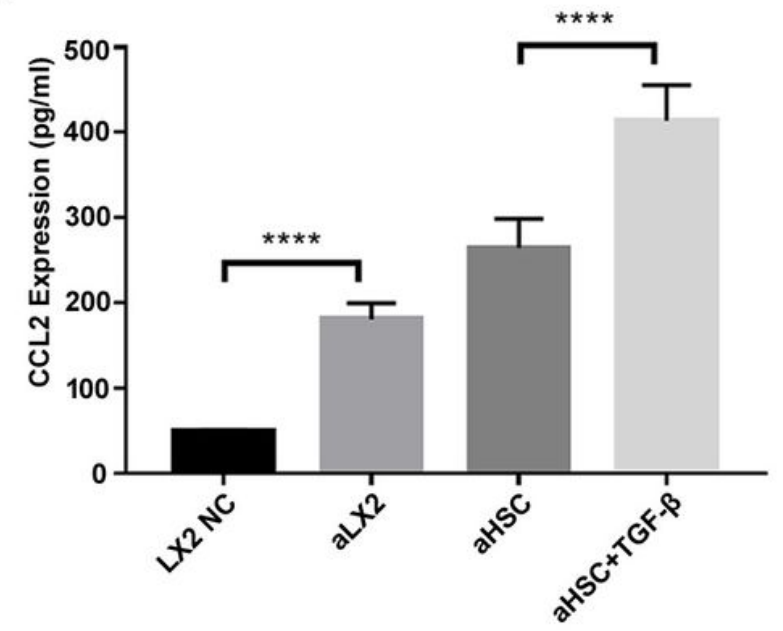

C

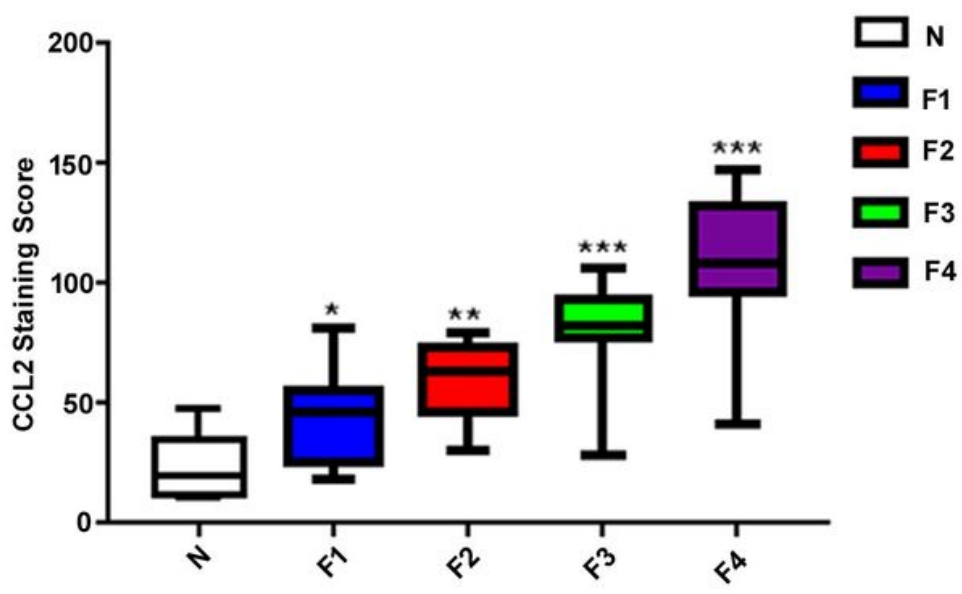

D

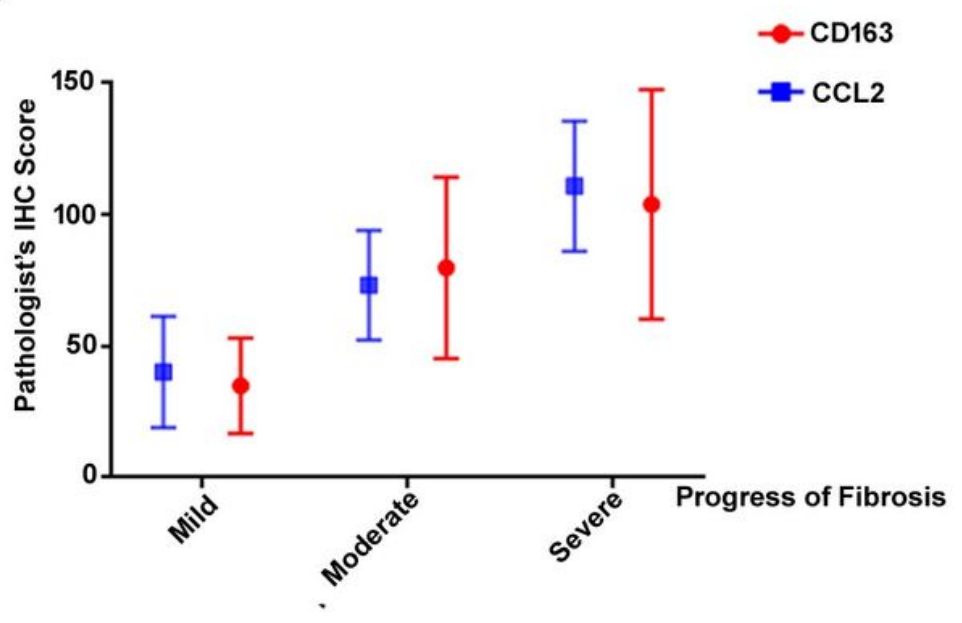

Figure 3

AHSCs secret high levels of CCL2. As was shown in Figure 3A, the primary aHSCs are typically fusiform, and express the activation marker a-SMA, together with a high expression of CCL2 protein. In Figure 3B, aHSCs can secrete high levels of CCL2 compared with LX2, and interestingly this ability can be further enhanced with stronger activation induced by TGF- $\beta$ (LX2 vs. TGF- $\beta$ stimulated LX2, $P<0.001$; aHSC vs. TGF- $\beta$ stimulated aHSCs, $P<0.001)$. Besides, as the degree of liver fibrosis worsen, the expression of CCL2 gradually increased compared to the control group shown in Figure 3C, (CCL2 staining score of $\mathrm{N}$ is 23.26 \pm 13.85; F1: $48.56 \pm$ 19.18, P=0.03; F2: $58.25 \pm$ 16.24, P <0.001; F3: $81.33 \pm 18.48, P<0.001 ; F 4: 110.93 \pm$ 24.75, $\mathrm{P}<0.001$. Also, there was a strong relationship between M2 macrophage (CD163+) IHC score and CCL2 IHC score. The CCL2 IHC score in the mild degree was $37.9 \pm 19.96$; moderate was $72.76 \pm 20.60$; severe was $110.92 \pm 24.64$, as is shown in Figure 3D. 
A
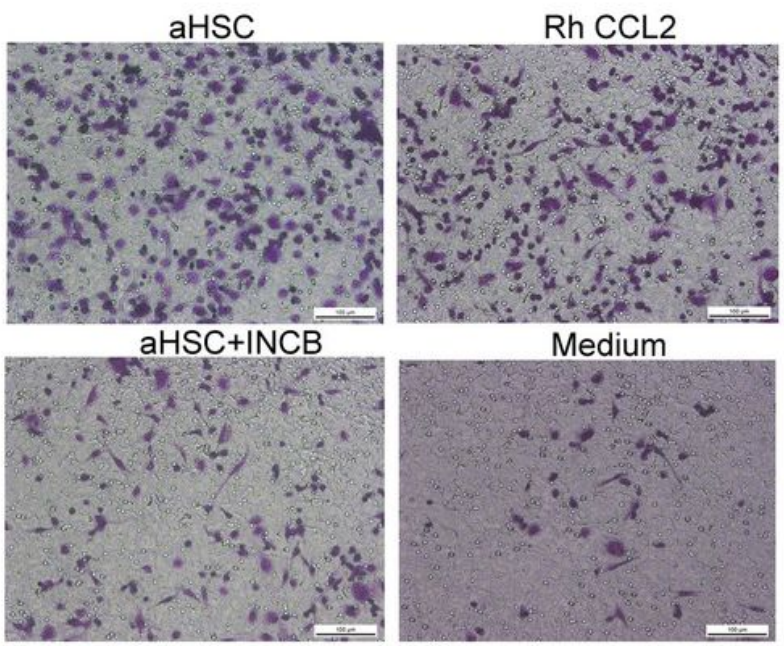

Medium

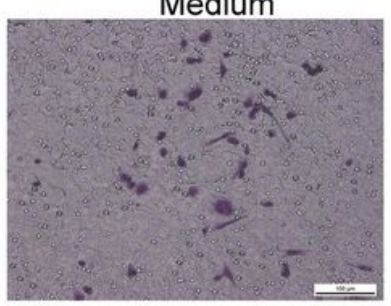

B

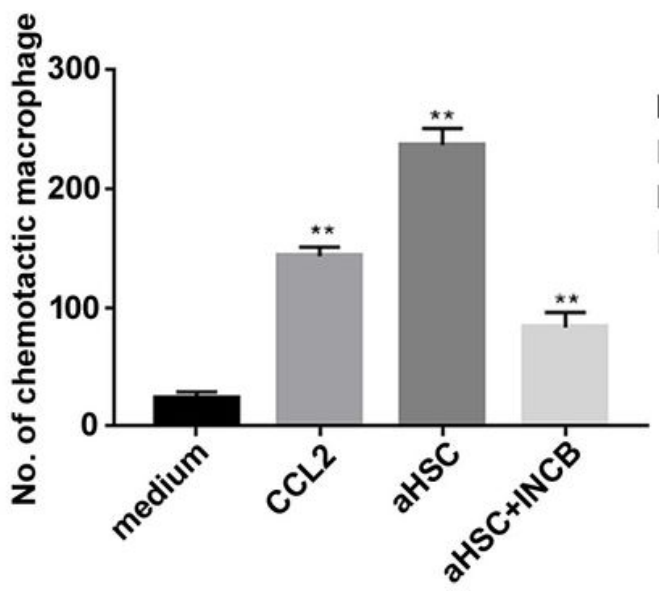

C

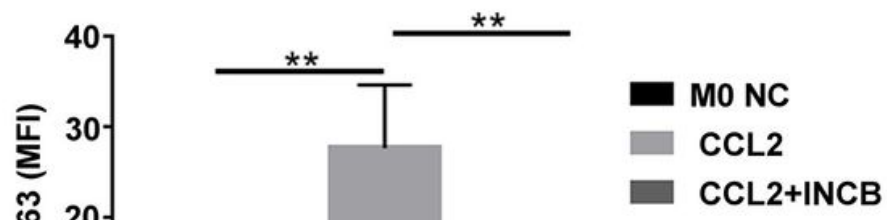

D

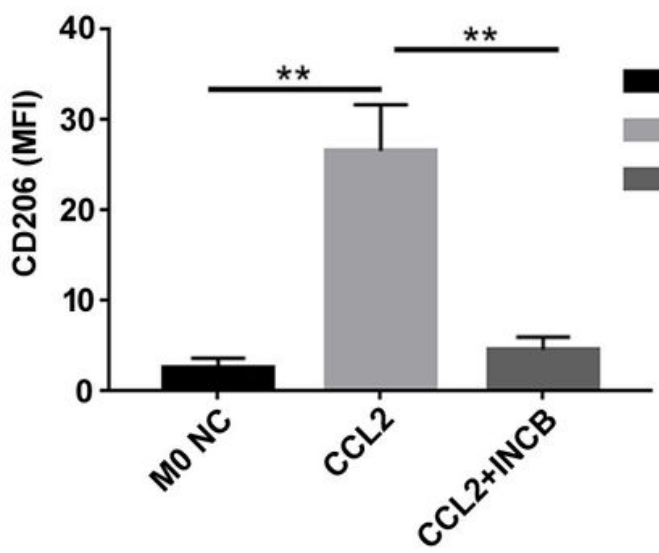

Figure 4

AHSCs induce macrophage infiltration and M2 differentiation via CCL2. A showed the macrophage infiltration significantly increased when aHSCs were cultured in the lower chamber compared to control (only medium), and the number decreased while we added CCL2 specific receptor antagonist INCB to block CCL2/CCR2 pathway in the same culture system. B showed a paired t-test between each group and the control group, and ** indicates $P<0.01$. C-D showed CD163 and CD206 expression significantly upregulated on macrophages under the stimulation of Rh CCL2 compared to the MO NC control group, CD163: $27.6 \pm 7.0 \%$ vs $2.7 \pm 1.1 \%, P=0.008 ; C D 206: 26.5 \pm 5.1 \%$ vs $2.4 \pm 1.2 \%, P=0.003$. The addition of INCB $(100 \mathrm{ng} / \mathrm{mL})$ prohibits the expression of CD163 and CD206 on the MOMФ (CD163: $4.5 \pm 1.4 \%$, CD206: $4.1 \pm 2.6 \%$, vs M0 NC group, $P>0.05$ ). 
A

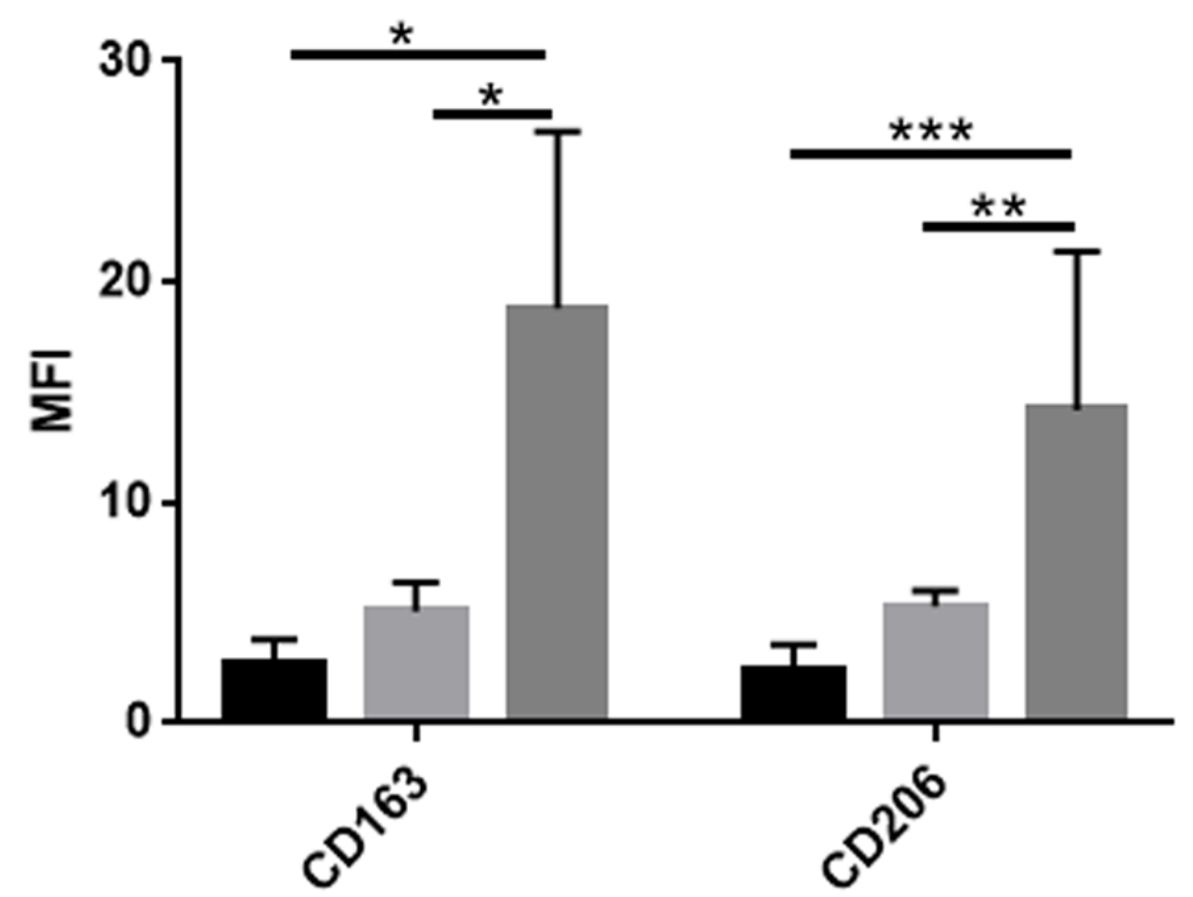

$\square \mathrm{MONC}$

LX2 supernant

aLX2 supernatant

B

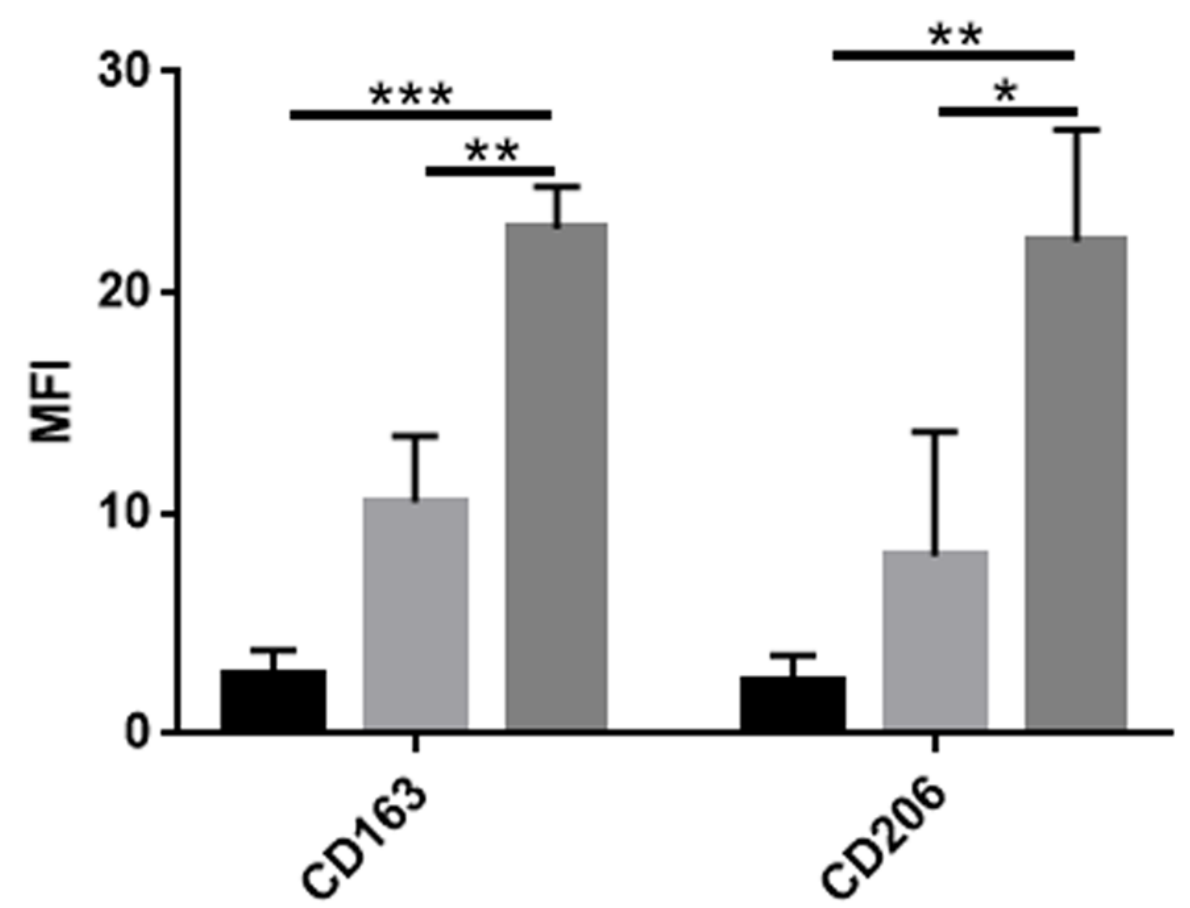

MO NC

CO-LX2

CO-aLX2

Figure 5

LX2 activated under the stimulation of TGF- $\beta$ could upregulate the expression of CD163 and CD206 on macrophages both in the co-culture system and in supernatant stimulation way. In the supernatant treatment, the expression of CD163 and CD206 in the aLX2 group, compared to the LX2 group, was respectively: $18.8 \pm 8.0 \%$ vs $5.1 \pm 1.3 \%, P=0.007$, and $14.2 \pm 7.2 \%$ vs. $5.3 \pm 0.7 \%, P=0.048$. The expression of 
CD163 and CD206 in co-aLX2 group was significantly higher than that in the co-LX2 group: $22.9 \pm 1.9 \%$ vs. $10.5 \pm 3.0 \%, P<0.05 ; 22.3 \pm 5.1 \%$ vs. $8.1 \pm 5.6 \%, P<0.05$.

A

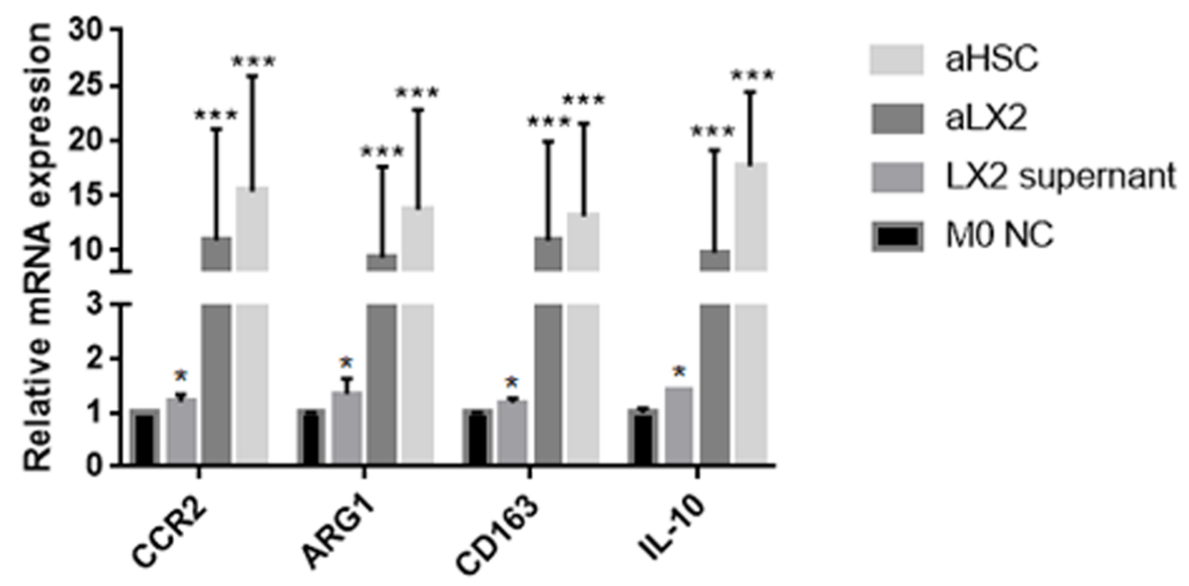

B

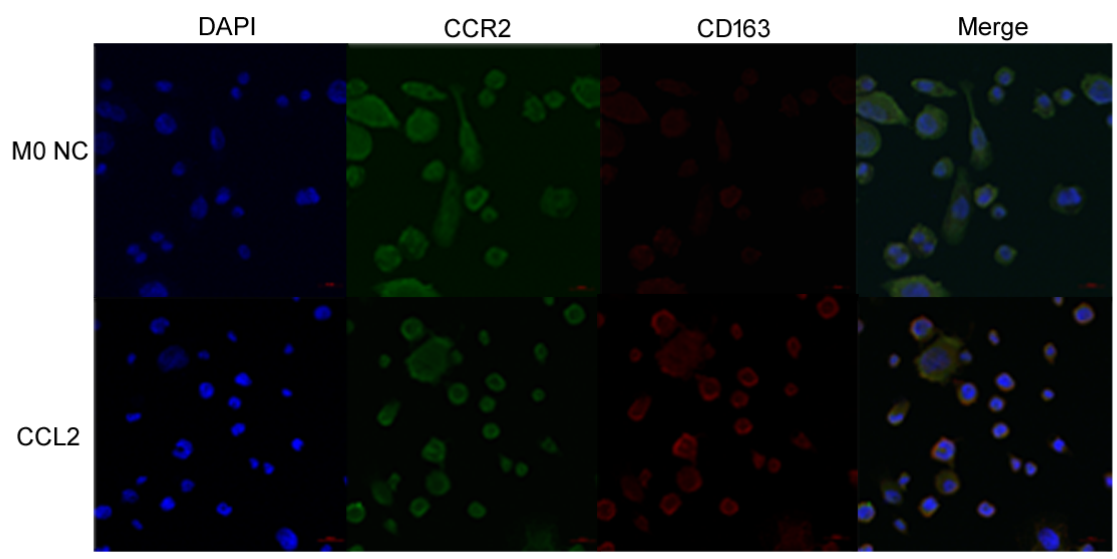

C

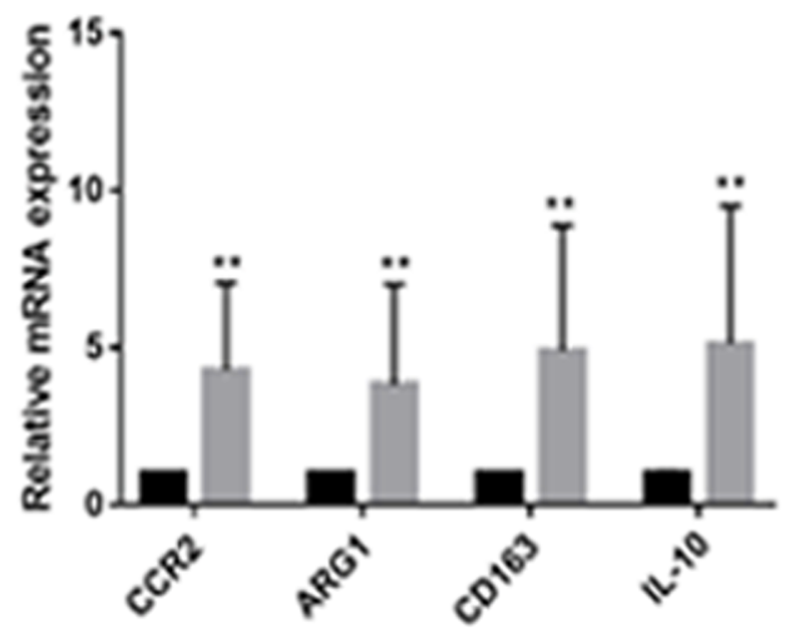

MONC

Din $\operatorname{COCl} 2$

\section{Figure 6}

AHSCs upregulated macrophage CCR2 expression to form CCL2/CCR2 positive feedback circle pathway. We tested M2MФ specific markers expression in mRNA levels after treating THP-1 derived M0MФ at different conditions (supernatants stimulation from aHSC, aLX2, LX2, and complete medium with or 
without containing $5 \mathrm{ng} / \mathrm{mL}$ CCL2 for 5.5 days) by qPCR. A and $\mathrm{C}$ showed CCL2 could upregulate macrophage CCR2 expression besides CD163, ARG-1, IL-10 at the gene level. As was shown in B, the upregulation of both CD163 and CCR2 on CCL2 stimulated macrophages by immunofluorescence. shows that the Rh CCL2 group can significantly upregulate MФ's ability to express M2-type specific markers. * indicates $\mathrm{P}<0.05$, ** indicates $\mathrm{P}<0.01$, and $* \star *$ indicates $\mathrm{P}<0.001$.

\section{Supplementary Files}

This is a list of supplementary files associated with this preprint. Click to download.

- Supplementarymaterial.docx 